\title{
Gamificação e seus potenciais como estratégia pedagógica no Ensino Superior
}

\section{Cristina Martins ${ }^{1}$, Lucia Maria Martins Giraffa ${ }^{2}$, Valderez Marina do Rosário Lima $^{3}$}

\author{
1 Doutoranda em Educação pela PUCRS, bolsista CAPES/PROEX \\ ${ }^{2}$ Professora Titular - Senior Lecture Computer Science School- FACIN/PUCRS e \\ Professora pesquisadora da Escola de Humanidades-PPGEdu/PUCRS. \\ ${ }^{3}$ Professora permanente Escola de Humanidades-PPGEdu/PPGEducem-PUCRS.
}

Resumo: A sociedade contemporânea é fortemente influenciada pela cultura digital, os jogos digitais estão imbricados aos hábitos sociais e a gamificação emerge como elemento para o cotidiano dos espaços formais de educação. Gamificar é o uso de elementos de jogos digitais em atividades que não são jogos, no contexto educacional defendemos esta prática como uma estratégia pedagógica que está resgatando aspectos da ludicidade de experimentações relacionadas a metodologias e a didáticas ativas. Logo, nosso objetivo foi investigar o uso da gamificação como estratégia pedagógica no Ensino Superior, buscando indícios de potenciais pedagógicos. Esta é uma investigação qualitativa, apoiada em estudo de caso, com um grupo de 18 sujeitos de pesquisa, estudantes de cursos de Pós-Graduação stricto sensu. Realizamos um experimento empírico para que os sujeitos de pesquisa pudessem vivenciar uma prática pedagógica baseada na estratégia de gamificação e avaliar esta prática com base numa ficha avaliativa, com variáveis que pudessem nos indicar seus possíveis potenciais pedagógicos. Os resultados apontam que a gamificação apresenta potencialidades pedagógicas associadas aos processos de ensino e aprendizagem de professores e estudantes, e um alinhamento da com as vivências da cultura digital.

Palavras-chave: Gamificação, Estratégia Pedagógica, Ensino Superior.

\section{Gamification and potential as a pedagogical strategy in Higher Education}

\begin{abstract}
Contemporary society is strongly influenced by digital culture, digital games is part with social habits and gamification emerges as an element in the daily life of formal educational spaces. Gamify is the use of elements of digital games in activities that are not games, in the educational context we defend this practice as a pedagogical strategy that is recovering aspects of the playfulness of experimentations related to methodologies and active didactics. Therefore, the aim was investigated the use of gamification as a pedagogical strategy in Higher Education, understand of pedagogical potentials. This is a qualitative research, supported by a case study, with a group of 18 research subjects, graduate students. We performed an empirical experiment that the research subjects could experience a pedagogical practice based gamification strategy and evaluate this practice based on a form with variables that could indicate potential pedagogical. The results show that the gamification presents pedagogical potential associated with the teaching and learning processes of teachers and students and an alignment of the experiences of the digital culture.
\end{abstract}

Keywords: Gamification, Pedagogical Strategy, Higher Education.

\section{INTRODUÇÃO}

Na sociedade contemporânea, o ciberespaço possibilita (re) invenção de práticas sociais e culturais humanas, ampliando funções cognitivas, condicionando 
comportamentos, hábitos e atitudes, como já postulava Lévy (1999). Estamos conectados e interagindo com outras pessoas em qualquer momento e lugar, cada vez mais com facilidades de acesso e de recursos prestando serviços para atender nossas necessidades cotidianas básicas. Estas evoluções rápidas e massivas advindas das tecnologias digitais e seus serviços repercutem na ideia de escola/universidade, na concepção de aula presencial, no espaço de ensino formal e no fazer dos estudantes e professores. Neste emaranhado de fios simbólicos, docentes e estudantes ressignificam papéis e criam novas tessituras para dar significado aos movimentos de ir e vir partindo/chegando do presencial ao virtual.

Neste cenário de cibercultura, os jogos, principalmente os digitais, se apresentam fortemente ligados aos hábitos sociais cotidianos, influenciados pelo uso (quase) massivo de dispositivos móveis. Neste contexto midiático, plural e com fortes componentes lúdicos a gamificação emerge como elemento para o cotidiano dos espaços de educação formal. Gamificar, prática idealizada por Jane McGonigal (2011), é usar elementos típicos das dinâmicas de jogos para desenvolver atividades que não são jogos. Um dos principais motes para gamificar uma prática pedagógica é alcançar graus mais elevados na motivação da aprendizagem e engajamento dos estudantes, por exemplo a partir de resoluções de problemas.

Neste artigo, abordamos a gamificação como uma estratégia pedagógica usando elementos típicos dos RPG (Role-playing game), modalidade de jogo em que os jogadores assumem papéis associados a personagens e criam narrativas colaborativamente. Para isso, inicialmente apresentaremos reflexões sobre possíveis análises de estratégias de ensino e de aprendizagem, abordando formas de reconhecê-las como pertinentes à luz de nossos referenciais, o que nos permite avaliar os potenciais pedagógicos. Cabe ressaltar que entendemos que os potenciais pedagógicos são um conjunto de características presentes em uma estratégia, que impactam de maneira promissora os processos de ensino e de aprendizagem. A estratégia pedagógica é a definição de objetivos e metas e das escolhas teóricas e metodológicas possíveis nas ações ou sequências didáticas que envolvem os processos de ensino e de aprendizagem. Colocamos o adjetivo "pedagógico", pois defendemos que uma estratégia deve articular e organizar as condições de ensino para que possa se construir aprendizagens concretas, ou seja, considerando além dos processos de ensinar, também os processos de aprender.

Sendo assim, desenvolvemos uma investigação com abordagem de cunho qualitativo, em que a questão norteadora que conduziu a discussão foi: quais as potencialidades pedagógicas do uso da estratégia gamificada no Ensino Superior? Para dar conta deste questionamento, tivemos como objetivo: investigar o uso da gamificação como estratégia pedagógica no Ensino Superior, a partir de um estudo de caso, buscando indícios de potenciais pedagógicos.

\section{UM ENFOQUE POSSÍVEL NA ANÁliSE DE ESTRATÉGIAS PEDAGÓGICAS}

Inicialmente, nossa discussão se organizará em torno de identificar elementos teóricos que nos permitam indicar um conjunto de características presentes em uma estratégia pedagógica para reconhecê-las como apropriadas, permitindo-nos avaliar potenciais pedagógicos. Alguns autores nos dão subsídios para discutir estes elementos, os quais podemos considerar como de maior relevância ao optar por uma estratégia pedagógica. Zabala (2003) diz que os critérios que podem indicar a pertinência educativa de uma sequência didática devem abordar o que se pretende fazer e os meios 
que podem facilitar a consecução desse objetivo. Logo, o autor defende como instrumentos para analisar a prática docente:

- a discriminação tipológica dos conteúdos de aprendizagem, podendo estes serem de natureza conceitual, procedimental ou atitudinal e suas relações com as intenções educativas e coerência entre o que se diz e o que se faz.

- a percepção sobre o ensino e a função social atribuída aos processos de aprender e ensinar, sustentada por princípios construtivistas, que compõem uma prática epistemologicamente pautada no interacionismo.

Vianna (2014) postula que questões relacionadas ao emprego dos instrumentos de medida em avaliação educacional devem ser dimensionadas a fim de que os resultados façam sentido e permitam a orientação das atividades docentes. Para tanto, os resultados das avaliações não devem ser usados apenas para traduzir um certo desempenho escolar, mas para a definição de elementos para a tomada de decisões, que visem provocar mudanças no pensar e no agir dos docentes. Então, Vianna $(1976,2014)$ defende a ideia de que a medida da qualidade em educação não se restringe somente desempenho escolar, é preciso se balizar, também, em outras variáveis que se associam e condicionam o rendimento escolar. Ele define princípios sobre a importância de ter um aporte em medidas de qualidade educacional: (a) é fundamental para uma educação eficiente; (b) os instrumentos de medida facilitam as observações que o professor faz do desempenho do aluno; (c) todos os objetivos educacionais importantes podem ser analisados e avaliados quantitativamente e qualitativamente. Cabe destacar que para o autor, o processo de medida não visa somente a obtenção de escores numéricos, mas analisar as situações relativas à aprendizagem ocorridas através do ensino, às respostas dos indivíduos as situações apresentadas e à classificação dos indivíduos segundo suas respostas. (VIANNA, 1976).

Entendemos que quando decidimos sobre uma determinada estratégia pedagógica, é preciso considerar o que e como estão sendo propostas ao estudante, bem como oferecer possibilidades contextualizadas com seus hábitos cotidianos, ou seja, alinhada com suas expectativas que se relacionam com a realidade vivida fora do ambiente do ensino formal, a partir de problemáticas cotidianas. Por este motivo consideramos que a gamificação é uma alternativa frente as estratégias que possuem potenciais pedagógicos para impactar de maneira apropriada nos processos de ensino e de aprendizagem. Cabe ressaltar, que nossa intenção não é estabelecer critérios ou modos para identificar uma boa ou uma péssima estratégia pedagógica, pois estaríamos sendo reducionistas e simplistas. O que queremos discutir são as características ou as variáveis que nos fazem reconhecer potenciais pedagógicos em uma estratégia de ensino e de aprendizagem, sendo tal reflexão complexa e com múltiplas aberturas a novas discussões. Em suma, queremos prover de análise e reflexão sobre a prática pedagógica de modo a possibilitar novas compreensões sobre processos de ensino e sua avaliação relacionada a pertinência educacional.

\section{O PORQUÊ DA GAMIFICAÇÃO}

Dentre as estratégias pedagógicas contextualizadas à condição sociocultural atual, a gamificação mostra-se como uma tendência promissora que pode ser considerada uma readaptação da cultura lúdica às técnicas condicionantes da cibercultura, um movimento natural imbricado ao desenvolvimento do ser humano por meio da interação com o lúdico. (MARTINS; GIRAFFA, 2015). Kapp (2012), que fez um estudo contextualizando a gamificação e a educação, afirma que gamificar contempla o uso de competências, mecânicas, estéticas e pensamentos dos jogos para 
engajar pessoas, motivar a ação, promover a aprendizagem e resolver problemas. Destaca que essa prática deve ser desenvolvida usando todos os elementos dos jogos digitais que forem apropriados ao contexto escolar, não apenas elementos que remetam a pontuação e recompensas. Logo, entendemos que gamificar uma atividade prática pedagógica não significa criar um jogo de viés pedagógico ou simplesmente jogar para ensinar. É preciso compreender e significar mecânicas e dinâmicas (elementos) presentes em jogos digitais, permeando-os em práticas pedagógicas.

Schlemmer (2014) destaca que a gamificação na educação consiste em aplicar a forma de pensar, os estilos e as estratégias que estão presentes em jogos digitais e os tornam divertidos; e para não cairmos em modismos, utilizando apenas elementos como: rankeamento, pontuação, classificação, etc.; devemos priorizar a aprendizagem a partir dos elementos de RPG digitais, que se mostram mais complexos e que podem levar a um maior engajamento e motivação, em atividades pedagógicas.

A percepção, de que elementos de jogos digitais que podemos aplicar ao gamificar uma atividade devem estar relacionados a uma prática pedagógica alinhada as bases construtivistas, de cunho sociointeracionista (VIGOTSKY, 2007), nos leva a considerar que nem todas as formas de gamificação discutidas atualmente são significativas a contextos educacionais. Dentre os elementos de jogos digitais, acreditamos serem significativos aqueles que desenvolvidos em atividades gamificadas, no contexto escolar, possam aprimorar competências relevantes ao estudante, tais como: colaboração, pensamento estratégico, cooperação, reflexão (pensamento crítico), autonomia, domínio de conteúdo, hábitos de estudo, etc.

Tendo em vista, este cenário teórico propusemos a construção de um modelo gamificado ${ }^{1}$ com intencionalidade educativa discutido em Martins e Giraffa (2015) e é apresentado na Figura 1, pois entendemos que é na combinação de elementos advindos do RPG digital e das correlações entre eles que produzimos uma experiência adequada à aprendizagem efetiva a partir do uso da gamificação.

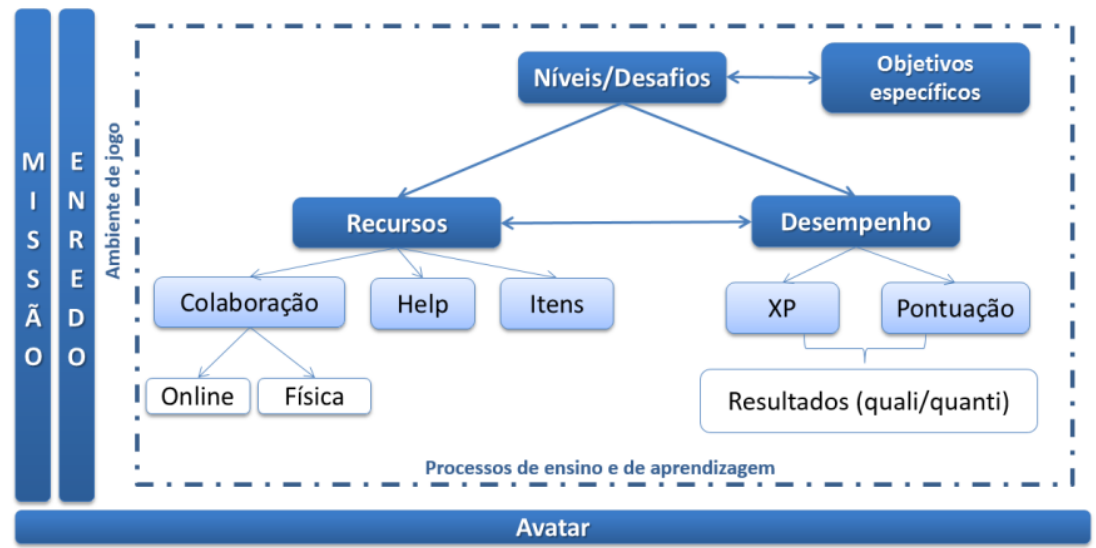

Figura 1 - Elementos de jogos digitais em atividades gamificadas.

Fonte: MARTINS; GIRAFFA, 2015.

Verificamos através dos nossos estudos que existem inúmeros elementos de jogos digitais, baseados nas mecânicas e dinâmicas de jogos, mas que nem todos valem um investimento educacional, por isso destacamos o RPG, que é um tipo de jogo diferenciado, em que a competitividade entre os jogadores não é o elemento central, há encenação de papéis diversos, contação de histórias, missões a serem cumpridas, articulação de estratégias, estabelecimento de relações com outros jogadores, mediação de conflitos e outros. Nesta proposição, para constituição de práticas pedagógicas gamificadas selecionamos os seguintes elementos de jogos digitais encontrados, 
principalmente em RPG digitais: missão, enredo, níveis/desafios, objetivos específicos, recursos, colaboração, help, itens, desempenho, xp, pontuação e avatar.

Então, buscando novas evidências para seguir avançando nas discussões sobre gamificação e suas interfaces educativas, agora, queremos abordar a discussão deste estudo no contexto do ensino superior, aprofundando o potencial pedagógico da gamificação como uma estratégia de ensino e de aprendizagem para com alunos universitários, como forma de atender os desafios educacionais que emergem do século XXI.

\section{PROCEDIMENTOS METODOLÓGICOS}

Realizamos uma investigação qualitativa baseada em estudo de caso (YIN, 2005). Como amostragem optamos por um grupo 18 sujeitos de pesquisa, estudantes de cursos de Pós-Graduação stricto sensu, de uma instituição de Ensino Superior privada, matriculados na disciplina Metodologia do Ensino Superior. Os estudantes são oriundos de cursos de Mestrado e Doutorado de diversas áreas do conhecimento. A disciplina Metodologia do Ensino Superior é obrigatória aos estudantes de cursos de Mestrado e Doutorado na instituição de ensino estudada e tem como ementa a reflexão crítica sobre o significado da educação, sobre os fundamentos da ação educativa e suas repercussões na definição de ensino e de aprendizagem a postulantes à docência no Ensino Superior. Desenvolver nossa investigação neste contexto e com esta amostra foi intencional pelo fato de que na disciplina é proposto aos estudantes o desenvolvimento de atividades de estudo e aplicação prática de estratégias pedagógicas, bem como são realizadas análises e reflexões destas estratégias por eles.

Realizamos um experimento empírico para que o grupo de estudantes (sujeitos de pesquisa) vivenciasse uma prática pedagógica baseada na estratégia de gamificação a partir da dinâmica da disciplina e avaliasse com base numa ficha avaliativa (instrumento de coleta de dados). Os estudantes foram divididos em pequenos grupos e para cada um foi atribuída uma estratégia pedagógica distinta. Neste trabalho em grupo, deveria ser realizado um estudo teórico e o desenvolvimento de uma prática pedagógica a ser aplicada com o restante da turma com base nas estratégias definidas pelas professoras. Cabe ressaltar que duas das autoras são professoras da disciplina vinculadas ao Programa de Pós-Graduação em Educação e uma das autoras é estudante do Doutorado em Educação, sendo estudante da disciplina e participante do grupo que foi atribuída a estratégia de gamificação. Contemplando aspectos éticos da pesquisa foi solicitada autorização para a coleta de dados e a divulgação dos resultados da investigação aos estudantes da turma, incluindo especialmente, os estudantes do grupo que ficou responsável por desenvolver o trabalho sobre gamificação junto com a autora-estudante.

O trabalho realizado pelo grupo de estudantes responsável pela estratégia de gamificação foi organizado a partir de uma sequência didática sobre a temática sustentabilidade, como segue: (a) organização dos estudantes da disciplina em grupos com 4 participantes; (b) aplicação da atividade gamificada proposta com o uso de elementos presentes em jogos de RPG digital, por meio de cartas, exemplificado na Figura 2; (c) análise das situações práticas resultantes da atividade, com identificação problemas e oportunidades, propondo soluções possíveis; (d) apresentação das conclusões de cada grupo a todos. Cabe ressaltar que ao planejar a atividade gamificada o grupo considerou o modelo gamificado com intencionalidade educativa descrito por Martins e Giraffa (2015) e apresentado na figura 1. 


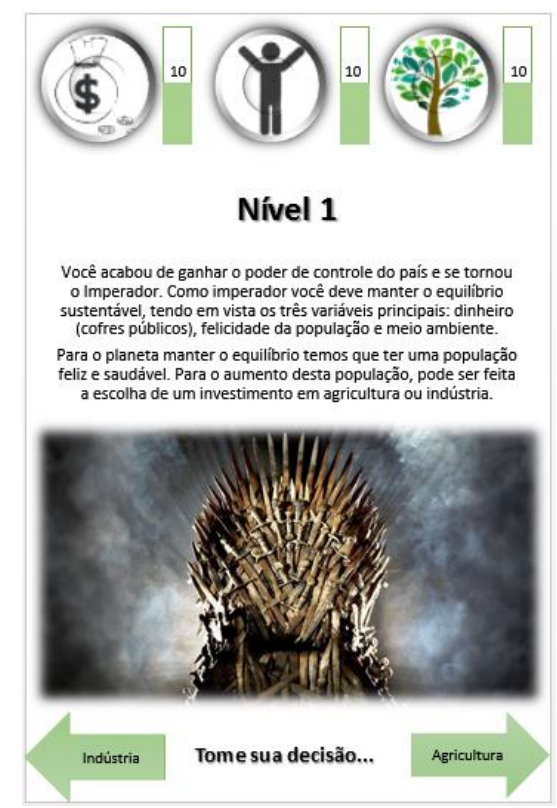

Figura 2 - Carta gamificada.

Fonte: Autoras, 2018.

Ao final da prática, foi aplicada com todos os estudantes da disciplina a ficha avaliativa, que foi um instrumento em forma de questionário fechado com itens relacionados à características ou à variáveis que auxiliam no reconhecimento de potenciais pedagógicos em uma estratégia de ensino e de aprendizagem, considerando os seguintes aspectos: planejamento do professor e desempenho do estudante. Os itens do questionário foram adaptados para a realidade atual a partir da discussão das medidas de qualidade educacional propostas por Vianna $(1976,2014)$. Consideramos aspectos relacionados a intervenções educativas propostos por Zabala (2003). O instrumento contou, também, com uma questão aberta sobre a aplicabilidade da estratégia pedagógica gamificação com estudantes do Ensino Superior.

$\mathrm{Na}$ análise dos dados a seguir, faz-se uma reflexão descritiva/comparativa com os itens propostos na ficha avaliativa respondidos de forma direta pelos estudantes e com as respostas abertas, a partir do método de análise de conteúdo proposto por Bardin (2009), organizado em três etapas: pré-análise, a exploração do material; e, por fim, o tratamento dos resultados: a inferência e a interpretação.

\section{DISCUTINDO AS POTENCIALIDADES DO USO PEDAGÓGICO DA GAMIFICAÇÃO COMO ESTRATÉGIA DE ENSINO}

Alinhar as estratégias pedagógicas em tempos de cibercultura implica considerar aspectos que desenvolvam a motivação e o engajamento dos estudantes. Seguindo ideais sociointeracionistas, que neste contexto se mostram adequados e podem sustentar metodologias ativas, indicamos aos sujeitos de pesquisa que analisassem a prática pedagógica baseada na estratégia gamificação a partir de duas variáveis: planejamento do professor e desempenho do estudante. Tecemos reflexões que constituem os resultados da investigação com bases nas indicações teóricas de Vianna $(1976,2014)$ e Zabala (2003).

Considerando potencialidades pedagógicas significativas ao contexto educacional, as duas variáveis foram subdividas em itens, conforme estrutura apresentada no Tabela 1. 
Tabela 1 - Elementos para análise de potenciais pedagógicos

\begin{tabular}{|c|c|}
\hline \multirow{2}{*}{ Planejamento do professor } & Objetivos \\
\cline { 2 - 2 } & Organização da aula \\
\cline { 2 - 2 } Desempenho do estudante & Seleção do material \\
\cline { 2 - 2 } & Participação do aluno \\
\cline { 2 - 2 } & Interação professor-aluno \\
& Fechamento \\
\hline
\end{tabular}

Fonte: Autoras, 2018.

No primeiro item de análise de potencial pedagógico foi questionado se ficou claro para os estudantes o objetivo da aula e seu percurso, os quais estavam relacionados ao planejamento do professor. Para 15 sujeitos de pesquisa este item foi contemplado com a aplicação da estratégia gamificada. Contudo, 3 sujeitos indicaram que o objetivo e o percurso ficaram em parte claros, sendo que este extrato de análise da questão aberta dá indícios desta percepção:

A ideia da atividade achei ótima, porém acredito que faltaram algumas orientações a respeito das instruções do game. Sujeito 1.

Lembramos que Zabala (2003) indica que é essencial aos estudantes compreenderem o que se pretende fazer e ao professor considerar os conhecimentos seus prévios. Neste caso, é possível compreender que nem todos os estudantes possuem conhecimentos relacionados a jogos digitais do tipo RPG, logo uma explanação partindo do pressuposto que nem todos são jogadores se faz necessária. Entretanto, isto não invalida a gamificação, mas é um fator de aprimoramento a ser considerado na aplicação desta prática.

No segundo item sobre a organização da aula, também, com relação ao planejamento do professor, foi perguntado se as etapas/atividades ofertadas aos estudantes foram definidas de modo a facilitar a aprendizagem. Neste caso 16 sujeitos indicaram que este aspecto foi contemplado e 2 indicaram foi contemplado em parte. Em relação a este potencial pedagógico apresentamos os extratos de análise:

Pode ser aplicado em aulas de clínica, essas aulas exigem raciocínio e conhecimento.

Podem também ser aplicado para qualquer faixa-etária, isso torna positiva a utilização. Recomendo o método. Sujeito 2

Eu usaria a estratégia, com certeza, pois há aprendizado de uma forma mais divertida e com mais interação do que uma aula comum. Sujeito 7

Quando bem planejada a gamificação pode ser uma forma criativa e eficaz, que envolve os alunos, gerando assim aprendizagem e reflexão. Sujeito 12

Diversos fatores foram apontados de maneira mais implícita ou até explícita, por meio da percepção dos sujeitos de pesquisa, os quais estão implicados a facilitação da aprendizagem durante a aplicação de uma sequência didática, tais como: raciocínio, conhecimento prático (saber-fazer), interação, dinamicidade por meio da diversão, criatividade, reflexão e envolvimento. Zabala (2003) diz que numa intervenção educativa com potencial pedagógico os conteúdos devem ser colocados de tal modo que sejam significativos e funcionais aos estudantes, provocando conflitos cognoscitivos e promovendo a atividade mental. Considerando este aporte articulado aos fatores de facilitação da aprendizagem foi possível evidenciar que alcançamos com a prática gamificada diferentes tipos de conteúdo (saber, saber-fazer, saber-ser), como proposto na perspectiva das competências, indo além de apenas conteúdos conceituais.

Em relação ao terceiro item, seleção de materiais, perguntamos se o material apresentado considerou experiências prévias dos estudantes. Foram 13 respostas positivas e 5 respostas indicando que contemplou em parte. Entendemos que os estudantes não jogadores necessitam de uma explanação maior sobre o funcionamento da atividade gamificada, assim como apareceu no primeiro item de análise, o que 
reforça nossa reflexão sobre o aprimoramento de práticas gamificadas. Os extratos de análise que fazem relação a este item remetem, também, a preocupação quanto aos conhecimentos sobre jogos dos professores que planejam uma prática gamificada:

Achei a proposta muito boa e sim, usaria com estudantes do Ensino Superior. Contudo o tema/assunto/conteúdo pode apresentar dificuldades na elaboração das cartinhas. A lógica é muito legal e envolve os estudantes. Sujeito 6

Eu gostaria muito de utilizar, porém requer muito planejamento e tempo para executar, e dependendo da carga-horária do professor complica. Sujeito 11

A preparação/criação do jogo parece demandar muito esforço e o conhecimento prévio de jogos que existem no mercado, nos quais se basear. Sujeito 13

Zabala (2003) fala sobre o papel do professor nas interações educativas, indicando que o professor está numa posição de mediador entre aluno e cultura, o que exigirá dele desafiar-se. Faz parte do papel do professor aprofundar-se nas diversas possibilidades estratégias pedagógicas alinhadas com o contexto sociocultural. Com a gamificação não é diferente, ela abre espaço de experimentação ao professor, que ao buscar a compreensão sobre jogos digitais, estará mobilizando novos saberes docentes, os quais podem causar insegurança num momento inicial. Mas este movimento faz parte da construção de uma prática que venha a ter potenciais pedagógicos. Isso, também, converge ao que Kapp (2012) diz sobre a gamificação quando afirma que é uma estratégia educacional complexa de implementar.

No quarto item de análise de potencial pedagógico, passamos a perguntar sobre o desempenho do estudante durante a estratégia, considerando a abertura de participação (envolvimento) e o tempo para que pudessem pensar e reorganizar ideias face aos desafios apresentados. Todos os sujeitos de pesquisa indicaram que a estratégia contemplou este item. Logo, concordamos com Zabala (2003) as estratégias de ensino e de aprendizagem pertinentes nos tempos atuais devem ajudar a fazer com que os alunos adquiram destrezas relacionadas com aprender a aprender e que permitam ser cada vez mais autônomos em suas aprendizagens. Prover do envolvimento do estudante e de tempo para os diferentes ritmos de aprender apoiam uma prática ativa e podem ser desenvolvidos por meio de atividades gamificadas. Alguns extratos de análise corroboram nossa reflexão:

A proposta pode ser aplicada em qualquer área para qualquer conteúdo trabalhado. Achei ótima a estratégia, permite a reflexão, o posicionamento do aluno sobre o tema, leva em consideração os conhecimentos que o estudante já possui. Sujeito 3

É uma atividade que contempla a interatividade e o poder de escolhas dos estudantes, motivando-os para o trabalho. Este é útil quando os conteúdos a serem trabalhados são mais rígidos, assim são trabalhados com maior interesse. Sujeito 9

O quinto item do instrumento versa sobre a abertura a interação professor-aluno expressa na estratégia pedagógica, em relação ao desempenho do estudante. Aqui 16 sujeitos indicaram que o item possibilita esta interação e 2 sujeitos indicaram que possibilita em parte. Zabala (2003) estabele que a pertinência da estratégia de ensino e de aprendizagem a qual o professor opta deve fomentar atitudes favoráveis, motivando os estudantes a aprendizagem de novos conteúdos. A interação aluno-professor influi nesta variável, sendo um fator importante de ser desenvolvido. A gamificação tem como mote engajar e motivar o estudante em suas aprendizagens e percebemos este potencial por meio das representações dos sujeitos de pesquisa. Apresentamos um extrato de análise em que o sujeito demostra tal percepção em relação a gamificação:

A gamificação propicia um ambiente de troca, e possui uma dinâmica interessante para a problematização de temas. Nessa "estratégia de ensino" as "regras do jogo" devem ser claras. Eu pude perceber pela aula que tivemos como é importante apresentar a atividade previamente para manter os alunos engajados durante o exercício. Sujeito 5

No último item de análise do potencial pedagógico da estratégia de aprendizagem perguntamos sobre o fechamento da atividade gamificada articulado ao 
desempenho do estudante, considerando a consolidação de conceitos, procedimentos e atitudes fundamentais sobre o conteúdo e demonstração destas aprendizagens. Para esta pergunta 15 sujeitos responderam que sim, levou em consideração os itens perguntados e 3 sujeitos responderam que foram considerados em parte. Zabala (2003) diz que o potencial pedagógico de uma intervenção educativa tem relação com promover atividade mental que possibilite ao estudante estabelecer relações, generalizar e a atuar de forma autônoma, ou seja, ter um papel ativo, resultando na construção de aprendizagens. Evidenciamos que a gamificação pode permitir ao estudante protagonizar aprendizagens, dependendo da forma como for aplicada em uma prática pedagógica. Os sujeitos de pesquisa expressaram nos seus depoimentos escritos indícios que nos levaram a constatar esta potencialidade na prática gamificada que vivenciaram:

Utilizaria a estratégia especialmente por sua capacidade de promover um entendimento complexo do modo cada ação e escolha tem consequências que podem ser mais ou menos previstas. Também o aspecto da tomada de decisões conjunta parece ser mais facilmente trabalhado nesta estratégia, já que os debates são necessários à continuidade do jogo e o aspecto lúdico reduz eventuais tensões inerentes à interação entre pontos de vista conflitantes. Penso que o uso do jogo estimula o raciocínio em termos de solução de problemas, e também, pode ser suporte ao desenvolvimento da criatividade dos alunos. Sujeito 8

A atividade contribui para a reflexão, debate e solução de problemas a respeito de assuntos que venham a ser apresentados pelo professor. $O$ que permite uma análise reflexiva sobre as decisões que tomamos e o quanto elas tem relação com o que nos constitui. Sujeito 15

Para análise e reflexão das características, adaptamos as medidas de qualidade educacional proposta Vianna $(1976,2014)$, selecionamos as que consideramos que deveriam estar presentes em uma estratégia pedagógica, pois impactariam de maneira promissora nos processos de ensino e de aprendizagem dos estudantes e nos apoiariam a identificar potenciais pedagógicos que podem ser contemplados em uma prática gamificada. Desta forma, constatamos que a gamificação é uma estratégia pedagógica com potencialidades para ser aplicação em contextos de Ensino Superior.

\section{CONSIDERAÇÕES FINAIS}

Foi nossa intenção identificar elementos teóricos que nos permitissem indicar um conjunto de características presentes em uma estratégia pedagógica para reconhecêla como apropriada, permitindo a avaliação de potenciais pedagógicos. Discutimos a gamificação, pois se mostra como uma estratégia alinhada à cibercultura, em que os hábitos cotidianos estão fortemente vinculados as mídias e componentes lúdicos associados as tecnologias digitais e seus serviços. Além disso, era nosso objetivo avançar na discussão da gamificação em contextos educacionais, neste caso, em especial o Ensino Superior.

Refletir sobre as características ou variáveis que nos fazem reconhecer potenciais pedagógicos em uma estratégia de ensino e de aprendizagem é algo complexo e com múltiplas aberturas a novas discussões, portanto nosso estudo não é estanque. Logo, problematizamos os achados sobre o experimento prático com estudantes de Pós-Graduação stricto sensu, possibilitando diferentes compreensões sobre processos de ensino e a avaliação relacionada a pertinência educacional.

Para responder a nossa questão norteadora, pautando-se em nossos referenciais, indicamos que a gamificação apresentou as seguintes potencialidades pedagógicas:

- auxiliar na facilitação da aprendizagem pois desenvolve: raciocínio, conhecimento prático (saber-fazer), interação, dinamicidade por meio da diversão, criatividade, reflexão, envolvimento.

- desenvolver diferentes tipos de conteúdo de aprendizagem (saber, saberfazer, saber-ser). 
- exigir do professor a criação de espaços de experimentação, desafiando-o a construir novos saberes docentes.

- engajar o estudante e atender a diferentes ritmos de aprender, logo se configura como uma metodologia ativa.

- promover a interação aluno-professor, o que auxiliou no engajamento e na motivação do estudante em suas aprendizagens.

- possibilitar ao estudante estabelecer relações, generalizações e a atuações autônomas, ou seja, ter um papel ativo na construção de aprendizagens.

Cabe destacar que um achado importante relacionado aos objetivos, que se pretendeu desenvolver com a prática gamificada, foi considerar os conhecimentos prévios dos estudantes em relação a jogos digitais, nem todos os estudantes o possuíam. Logo, ao usar-se desta estratégia tal aspecto requer atenção do professor.

\section{NOTAS DE TEXTO}

${ }^{1} \mathrm{O}$ detalhamento deste modelo, bem como a seleção de elementos de jogos digitais que o compõe são apresentados no artigo "Gamificação nas práticas pedagógicas em tempos de cibercultura: proposta de elementos de jogos digitais em atividades gamificadas", das autoras Martins e Giraffa (2015), pode ser acessado no link: http://www.revistas.uneb.br/index.php/sjec/article/view/1236.

\section{REFERÊNCIAS}

BARDIN, L. Análise de Conteúdo. Lisboa, Portugal; Edições 70, 2009.

KAPP, Karl. The Gamification of Learning and Instruction: Game-based Methods and Strategies for Training and Education. San Francisco: Pfeiffer, 2012.

LÉVY, Pierre. Cibercultura. São Paulo: Editora 34, 1999.

MARTINS, Cristina; GIRAFFA, Lucia Maria Martins. Gamificação nas práticas pedagógicas em tempos de cibercultura: proposta de elementos de jogos digitais em atividades gamificadas. In: Anais do XI Seminário Jogos eletrônicos, educação e comunicação. Salvador: UNEB (Universidade do Estado da Bahia), 2015. Disponível em: <http://www.revistas.uneb.br/index.php/sjec/article/view/1236> Acesso em: 04/06/2016

MCGONIGAL, Jane. Reality is broken: why games make us better and how they can change the world. Nova York: The Penguin Press, 2011.

SCHLEMMER, Eliane. Gamificação em espaços de convivência híbridos e multimodais: design e cognição em discussão. Revista da FAEEBA - Educação e Contemporaneidade, Salvador, v. 23, n. 42, p. 73-89, jul/dez 2014.

VIANNA, Heraldo Marelim. Testes em educação. 1976.

VIANNA, Heraldo Marelim. Medida da qualidade em educação: apresentação de um modelo. Estudos em Avaliação Educacional, v. 25, n. 60, p. 36-42, 2014.

VIGOTSKY, Lev Semenovich. A formação social da mente: o desenvolvimento dos processos psicológicos. Org. por Michael Cole et al. 7 ed. São Paulo: Martins Fontes, 2007.

YIN, Robert K. Estudo de caso: planejamento e métodos. 3 ed. Porto Alegre: Bookman, 2005.

ZABALA, Antoni. Os enfoques didáticos. In: COLL, César; MARTÍN, Elena; MAURI, Teresa; MIRAS, Mariana; ONRUBIA, Javier; SOLÉ, Isabel; ZABALA, Antoni. O construtivismo em sala de aula. São Paulo: Ática, 2003. 